\title{
Ensino e aprendizagem no contexto do ensino remoto e da educação a distância: caminhos e possibilidades
}

\section{Teaching and learning in the context of Remote Education and Distance Learning: paths and possibilities}

\author{
Jorge Elias J $r^{1}$ (D), Fábio Carmona² (D), Mário Luís Ribeiro Cesaretti ${ }^{(D)}$, Valdes Roberto Bollela ${ }^{4}$ (D)
}

\begin{abstract}
RESUMO
Todo o conhecimento acumulado sobre o processo de ensino e aprendizagem, considerando as melhores práticas docentes e os conceitos sobre aprendizado de adultos, aprendizagem significativa e aprendizado centrado no estudante devem ser continuamente exercitados e revisitados, independente do formato: presencial, ensino remoto (ER) ou ensino a distância (EaD). A necessidade de ampliação da utilização do ER emergencial, determinada pela crise pandêmica de 2020, trouxe novos desafios para professores e estudantes em todo o mundo. Dentre os desafios se apresenta a reinterpretação dos conceitos presentes no processo de ensino e aprendizagem sob a lente do mundo digital. Esta revisão busca apresentar as melhores práticas sob o prisma do novo contexto da vida digital no ensino para as profissões da saúde, incluindo aqui as oportunidades oferecidas pela pandemia
\end{abstract}

PALAVRAS-ChAVE: Educação para profissões da saúde; Ensino remoto; Ensino à distância; Saúde digital; Covid-19

\begin{abstract}
All accumulated knowledge about the teaching and learning process, considering the best teaching practices and the concepts of adult learning, meaningful learning and student-centered learning must be continually exercised and revisited, regardless of the format: face-to-face, remote teaching (ER) or distance learning (EaD). The need to expand the use of emergency ER, determined by the pandemic crisis of 2020, has brought new challenges for teachers and students worldwide. Among the challenges is the reinterpretation of the concepts present in the teaching and learning process under the lens of the digital world. This review aims to present the best practices from the perspective of the new context of digital life in teaching for the health professions, including here the opportunities offered by the pandemic.
\end{abstract}

KEYWORDS: Health professional education; Remote education; Distance learning; Digital health; Covid-19

\footnotetext{
Docente do Departamento de Imagens Médicas, Hematologia e Oncologia Clínica da Faculdade de Medicina de Ribeirão Preto - USP.

Docente do Departamento de Puericultura e Pediatria da Faculdade de Medicina de Ribeirão Preto - USP.

Docente do Departamento de Clínica da Faculdade de Ciências Médicas e da Saúde - PUC-SP.

Docente do Departamento de Clínica Médica da Faculdade de Medicina de Ribeirão Preto - USP.
} 


\section{MENSAGENS PRINCIPAIS:}

- $\quad$ o processo de ensino e aprendizagem tem sofrido transformações para se adequar à era digital;

- A maioria dos conceitos básicos e fundamentais relativos ao processo de ensino e aprendizagem continuam válidos e contribuíram para formatar muitos dos recursos digitais;

- Novas competências relativas ao mundo digital são esperadas para estudantes, professores e profissionais em todos os campos do saber;

- Existem várias dicas e estratégias para planejamento e aplicação de atividades de ensino e aprendizagem no ER e EAD, buscando o aprendizado significativo do essencial

\section{INTRODUÇÃO}

O processo de ensino e aprendizagem tem sofrido profundas transformações determinadas pelas alterações do contexto histórico ao longo das últimas décadas, incluindo as revoluções, conflitos e pressão de demandas sociais e econômicas. Embora seja possível reconhecer vários marcos importantes nesta trilha de transformações, talvez um dos mais recentes e de maior impacto seja o advento da era digital. Nas últimas décadas pudemos assistir e participar da rápida evolução da comunicação por meio da internet, das mídias sociais e da indústria de hardware e softwares, cujo impacto em vários níveis é, e certamente continuará a ser por muito tempo, objeto de análise da história recente, considerando seu poder de transformar a sociedade e ao mesmo tempo ser transformado por ela.

Neste contexto, o ensino remoto (ER) e o ensino à distância ( $E a D$ ) também foram digitalmente transformados trazendo maior velocidade e dinamismo na comunicação, permitindo grande ampliação das ferramentas disponíveis para o desenvolvimento de planos de ensino-aprendizagem. A educação a distância, que se iniciou no século XVIII foi aquela que mais se aprimorou: se iniciou com postagens via correio, se aperfeiçoou com o advento das mídias (fita cassete, CD, DVD etc.) e atualmente está estruturada em plataformas online, com videoaulas gravadas, questionários online e atividades síncronas e assíncronas, além do contínuo desenvolvimento e lançamento de novos recursos instrucionais nas plataformas digitais. Atualmente existe um crescimento acelerado da oferta de cursos de graduação, pós graduação e educação continuada neste formato, bem como uma crescente utilização destas ferramentas na reestruturação de planos de ensino-aprendizagem constantes nos currículos das escolas, desde o ensino básico ao superior. A própria definição de ER e EaD deve ser vista com cautela ${ }^{1}$, considerando as transformações evolutivas que ocorreram nos últimos anos, embora seja consenso que EaD é uma modalidade de ensino, com modo de funcionamento próprio incluindo estruturação e desenho bem definidos até o modo avaliativo, enquanto o ER tem uma definição mais simples caracterizada por atividades e aulas que ocorrem através de veículos de transmissão de imagem e som, como a televisão, mas principalmente pela rede de internet. Ou seja, o EaD é um conceito mais amplo e mais detalhado, mas geralmente utiliza vários dos recursos de ER. Esta realidade foi exacerbada em 2020 com a pandemia SARS-Cov-2, que exigiu estratégias preventivas para controle da transmissão viral, como o isolamento social, a redução ou interrupção de atividades presenciais, evitando desta maneira aglomerações de pessoas e, consequentemente implicando na busca de soluções alternativas para manter o máximo possível das atividades de ensino ${ }^{2}$. No momento da pandemia, o ER denominado como "emergencial" foi utilizado como alternativa aprovada pelo MEC para manutenção das atividades na rede de ensino ${ }^{3}$. É interessante notar que a pandemia não afetou exclusivamente o ensino diretamente, mas também promoveu transformações na prática médica como um todo, apresentando desafio adicional na preparação das novas gerações de profissionais de saúde que deverão ter competências específicas para a prestação de cuidados e atendimento à distância ${ }^{2,4}$.

Por outro lado, é fundamental o entendimento de que todo o conhecimento acumulado e a melhor compreensão do processo de ensino e aprendizagem, em especial, para o aprendizado 
de adultos, compõem o arcabouço teórico e de melhores práticas que persiste válido para qualquer contexto. Deste modo, é oportuno revisitar este arcabouço e as melhores práticas sob o prisma do novo contexto da vida digital no ensino para as profissões da saúde, incluindo aqui as oportunidades oferecidas pela pandemia ${ }^{2}$. Este é o principal objetivo desta revisão.

\section{APRENDIZAGEM SIGNIFICATIVA DO ESSENCIAL}

Todas as profissões, incluindo as da área da saúde, têm definido seu conjunto de competências, as quais pressupõem a aquisição e desenvolvimento de habilidades cognitivas, psicomotoras e afetivas para o exercício profissional na sua plenitude. De modo geral, depreende-se que a aquisição e o desenvolvimento de tais habilidades sejam gradativos e escalonados, necessitando de ambientes educacionais adequados e de conjunto de situações-problema reais ou simuladas em número e complexidade adequadas para o momento específico do aluno na programação curricular.

Neste aspecto é fundamental que o professor tenha clara noção do seu papel na definição do conteúdo, elencando o que é realmente essencial quanto às habilidades esperadas para o aluno e deixando clara a intencionalidade das atividades direcionadas para seu desenvolvimento. Esta é uma das tarefas mais difíceis do professor, principalmente dos especialistas, qual seja a diferenciação do que é o essencial daquilo que é importante ou mesmo interessante. $O$ sucesso da aprendizagem significativa que impacta na formação de um bom profissional depende desta atuação do professor, independentemente do contexto: ensino presencial, ER ou EaD. Trata-se de um exercício constante, com risco perene do professor se desviar do essencial para aquilo que acredita que é importante ou interessante, passando a dar maior ênfase para aspectos periféricos do tema. Sem o planejamento adequado isto tende a ocorrer rapidamente, mas mesmo com planejamento este risco existe e pode determinar um desvio insidioso e imperceptível. Portanto, resta aqui a sugestão de revisão periódica da atuação docente, buscando prevenção da acomodação que a rotina oferece.
Muitos dos aspectos relacionados à aprendizagem de adultos, incluindo a motivação e interesse, bem como o conhecimento prévio do aprendiz e a disponibilidade de tempo dedicado às atividades propostas, são propícios para o desenvolvimento de habilidades cognitivas no ER e EaD, considerando tanto as ferramentas como as variações na aplicação destas atividades. Para isto é importante que a estruturação tenha o foco no estudante e permita flexibilidade no cumprimento das metas acordadas no desenvolvimento do conteúdo.

Embora exista uma percepção óbvia, bem como várias evidências, de que o ER não se configura como ambiente mais adequado para a proposição de atividades direcionadas para o desenvolvimento de algumas habilidades específicas, principalmente aquelas que requerem interação interpessoal, não se pode negar que houve algum avanço neste campo, especialmente no período de enfrentamento da pandemia. Questões como participação online, comportamento frente à câmera, preocupação em se fazer entender são alguns aspectos básicos que estão dentro do espectro das habilidades gerais para qualquer profissão na era digital 5 . Mas mesmo o profissionalismo, no seu aspecto mais amplo, pode e deve ser explorado nos ambientes virtuais, tanto nas atividades síncronas como nas assíncronas ${ }^{5-7}$.

Dentro do conjunto de preocupações para atingir a aprendizagem profunda ou significativa é fundamental que o essencial identificado pelo docente seja apresentado com contextualização direcionada, adequada e intencional, independente da estratégia educacional (presencial ou a distância, síncrona ou assíncrona), ou seja, perguntas como "porque aprender isso?", "qual será o impacto deste conhecimento na prática profissional?" e "em quais situações reais este conhecimento será necessário?" tanto auxiliam na definição do que é essencial quanto indicam "como" deve ser apresentado ao estudante. Assim, no planejamento e desenvolvimento de conjunto de atividades direcionado para tema essencial, a contextualização deve estar presente a todo momento, quer seja em aula teórica tradicional como em qualquer outra estratégia de ensino-aprendizagem. Além da contextualização, a estruturação do curso de forma a desafiar o estudante é funda- 
mental para aumentar a motivação do estudante para o aprendizado ${ }^{8}$.

O alinhamento entre a definição de um objetivo essencial (tema) de aprendizagem e sua aplicação através das estratégias de ensino-aprendizagem, culminam com a checagem do que foi aprendido pelo estudante, ou seja, a avaliação das competências pretendidas ${ }^{9}$. A(s) avaliação(ões) tem o poder de deixar claro o que é realmente essencial, e, portanto, tem o poder de direcionar o aprendizado, desde que o alinhamento esteja presente no planejamento geral. Deste modo, é óbvia a observação de que a avaliação sem o alinhamento adequado e planejado tem o poder inverso de confundir e desestimular os estudantes, com claro desperdício de tempo e de oportunidade de se alcançar momentos de aprendizado significativo. Os diversos tipos de avaliação são agrupados em avaliação somativa ou formativa, sendo que suas definições e meIhores indicações não são objetivo deste texto e podem ser encontradas em detalhe em outros $\operatorname{artigos}^{10-12}$. Porém, é importante salientar que existe grande discussão e esforço na busca de ferramentas que permitam maior segurança na realização de avaliações somativas à distância (online) ${ }^{13}$, enquanto há uma enorme facilitação para a realização de avaliações formativas no formato ER e EaD, incluindo mecanismos de devolutivas instantâneas, individualizadas por respostas e mais completas quando comparado ao formato presencial ${ }^{14-17}$

Por fim, são notórias as dificuldades que os formatos ER e EaD apresentam para o acompanhamento e engajamento dos estudantes. Existem várias estratégias descritas para minorar tais dificuldades, incluindo os aspectos gerais discutidos até aqui (definição do essencial, contextualização, alinhamento que inclui a avaliação), além de outras específicas, dentre as quais vale citar o envolvimento dos estudantes no planejamento, na medida do possível ${ }^{18}$.

Dentre os cuidados para um aprendizado mais efetivo estão as considerações e conceitos apresentados até aqui, os quais estão presentes na abordagem proposta por por Harden e Laidlaw na sigla em inglês "FAIR"19. A abordagem propõe que os alunos sejam tratados de maneira "justa" seguindo quatro princípios básicos (Quadro 1).
Quadro 1: 0 princípio "FAIR" para o aprendizado efetivo*

\begin{tabular}{lll}
\hline Acrônimo & Tradução & Descrição \\
\hline Feedback & Devolutiva & $\begin{array}{l}\text { Dê feedback aos alunos } \\
\text { à medida que eles pro- } \\
\text { gridem no domínio dos } \\
\text { resultados de aprendiza- } \\
\text { gem esperados }\end{array}$ \\
\hline Activity & Atividade & $\begin{array}{l}\text { Envolva o aluno na } \\
\text { aprendizagem ativa em } \\
\text { vez de passiva }\end{array}$ \\
\hline $\begin{array}{lll}\text { Individualisa- } \\
\text { tion }\end{array}$ & $\begin{array}{l}\text { Individuali- } \\
\text { zação }\end{array}$ & $\begin{array}{l}\text { Relacione o aprendizado } \\
\text { com as necessidades de } \\
\text { cada aluno }\end{array}$ \\
\hline Relevance & Relevância & $\begin{array}{l}\text { Torne o aprendizado } \\
\text { relevante para os alunos } \\
\text { em termos de seus ob- } \\
\text { jetivos profissionais }\end{array}$ \\
\hline
\end{tabular}

* Traduzido e modificado de Harden, Ronald M, Laidlaw, Jennifer M. Essential Skills for a Medical Teacher (p. 148). Elsevier Health Sciences

\section{CRIANDO OPORTUNIDADES DE APRENDIZAGEM}

Uma vez definido o que o estudante deve aprender, o passo seguinte está na definição de como ele vai aprender. Ou seja, quais oportunidades de aprendizagem serão oferecidas? Várias estratégias efetivas de ensino e aprendizagem foram desenvolvidas, testadas e comprovadas nas últimas décadas, compondo um conjunto de ferramentas à disposição dos professores. As características de cada ferramenta determinam sua melhor aplicação, considerando o propósito e a intencionalidade desejada para a aquisição ou desenvolvimento da habilidade elencada. Assim, aproveitando os componentes da pirâmide Miller ${ }^{20}$, onde na base temos o "saber" e o "saber como" relacionados aos conhecimentos essenciais e no ápice temos o "demonstrar" e o "fazer", portanto mais relacionados às habilidades específicas, podemos utilizar as "ferramentas" mais adequadas para alcançar a aprendizagem significativa desejada, lembrando sempre dos aspectos mais amplos relacionados às atitudes que são diretamente ligadas ao profissionalismo dentro do "ser, estar, e relacionar-se"12.

Assim, para cada objetivo de aprendizagem existe uma estratégia de ensino ou ferramen- 
ta (Quadro 2) mais adequada ${ }^{21}$. Além disso, um grande número de variações destas estratégias e ferramentas surgiram buscando uma maior adaptação para situações e objetivos particularizados.

Quadro 2: Estratégias e Ferramentas de Ensino e Aprendizagem*

Aprendizado baseado em problemas (PBL)

Aprendizado baseado em equipes ("Team-Based Learning" - TBL)

Ensino híbrido

Simulação

Ensino em ambientes profissionais - enfermarias, ambulatórios, comunidade

Estratégias diferenciadas de avaliação, como a formativa

*Modificado de Souza e cols. Estratégias inovadoras para métodos de ensino tradicionais - aspectos gerais. Medicina (Ribeirão Preto) 2014;47(3): 284-92

Sendo assim, na Era Digital, tais estratégias e ferramentas de ensino-aprendizagem foram de certa maneira sendo incorporadas ou, ainda, direcionaram o formato de vários recursos tecnológicos para o ensino, os quais, por sua vez, foram paulatinamente sendo consolidados como plataformas. Além da multiplicação da oferta de diferentes plataformas, verifica-se constantemente a atualização e disponibilização de novos recursos nas plataformas. O Ambiente Virtual de Aprendizagem (AVA) mais utilizado na Faculdade de Medicina de Ribeirão Preto (FMRP) é a plataforma de aprendizagem MOODLE, que é um recurso adotado institucionalmente pela Universidade de São Paulo, assim como a ferramenta G Suite for Education da plataforma Google. No entanto, existem várias outras plataformas disponíveis, inclusive várias versões do próprio Moodle ${ }^{22}$.

Tais plataformas permitem a utilização de muitas ferramentas úteis para comunicação, disponibilização de material, realização de reuniões online e aplicação de questionários e enquetes para verificação do aprendizado e discussão de dúvidas, para citar as mais utilizadas ${ }^{22}$. Destacase, porém, a necessidade de um planejamento e intencionalidade pedagógica para o uso correto das diferentes ferramentas tecnológicas ${ }^{23}$. A associação com outros softwares e serviços é pos- sível e permite grande flexibilidade para a escoIha do recurso mais adequado para determinada atividade. De modo geral, além da adequação do recurso à atividade ou objetivo elencado, a escoIha do que utilizar e quando é determinada por múltiplas características na visão do docente, do discente e da instituição (Quadro 3).

Quadro 3: Características que influenciam na escolha de recurso tecnológico para o ensino remoto Característica Considerações

Custo

Manutenção e supor- pouca ou nenhuma manute técnico tenção; baixo custo; suporte técnico acessível

Usabilidade amigável facilidade de instalação, utilização, aplicação; grau de investimento de tempo para atingir proficiência

Segurança mecanismos de proteção de propriedade intelectual; garantia de privacidade

Velocidade de acesso rapidez na instalação e na navegação

Interoperabilidade possibilidade de uso em comentre sistemas/plata- putadores, tablets e telefones formas celulares

Aceitação pelos do- a depender das demais caraccentes e pelos alunos terísticas pode ocorrer baixa adesão

Objetivo

solução completa ou parcial para desenvolvimento de uma determinada atividade

A descrição pormenorizada das múltiplas ferramentas e recursos tecnológicos para o ensino está fora do escopo deste texto, além de não ser tarefa que se esgote satisfatoriamente, dada à multiplicidade e variedade, bem como à velocidade acelerada de produção e atualização destes recursos. Ainda assim, cabe reconhecer que a partir de 2008, quando Andrew Churches revisitou a taxonomia de Bloom e Atherton para o mundo digital ${ }^{23}$, houve aumento do interesse em apresentar classificações dos recursos que permitissem melhor entendimento e aproveitamento de suas características na aplicação para o processo de ensino-aprendizagem ${ }^{25}$ (Figuras 1 e 2 ). 
Figura 1: A taxonomia digital de Bloom traduzida e adaptada de infográfico de Ron Carranza*

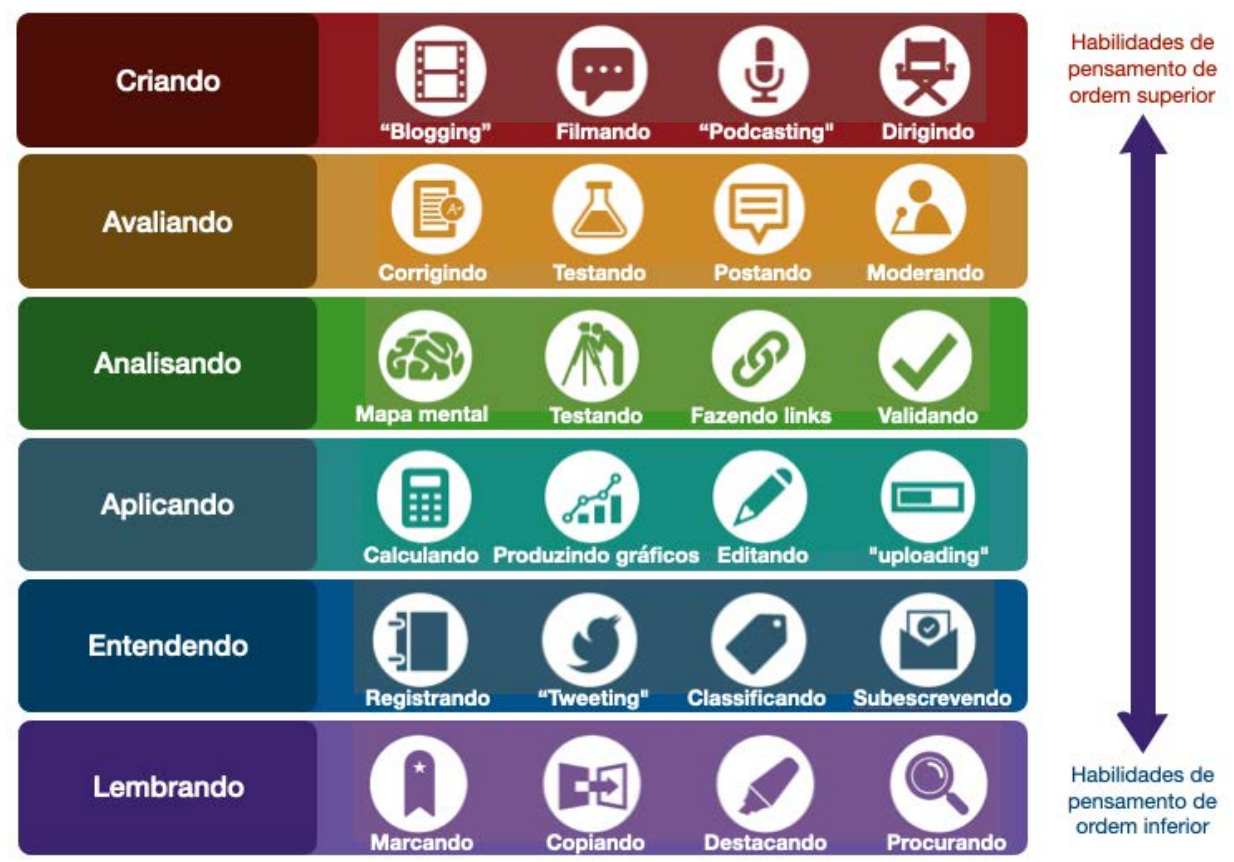

* https://teachonline.asu.edu/2016/05/integrating-technology-blooms-taxonomy/)

Figura 2: Pirâmide que associa várias tecnologias da web com a taxonomia de Bloom.

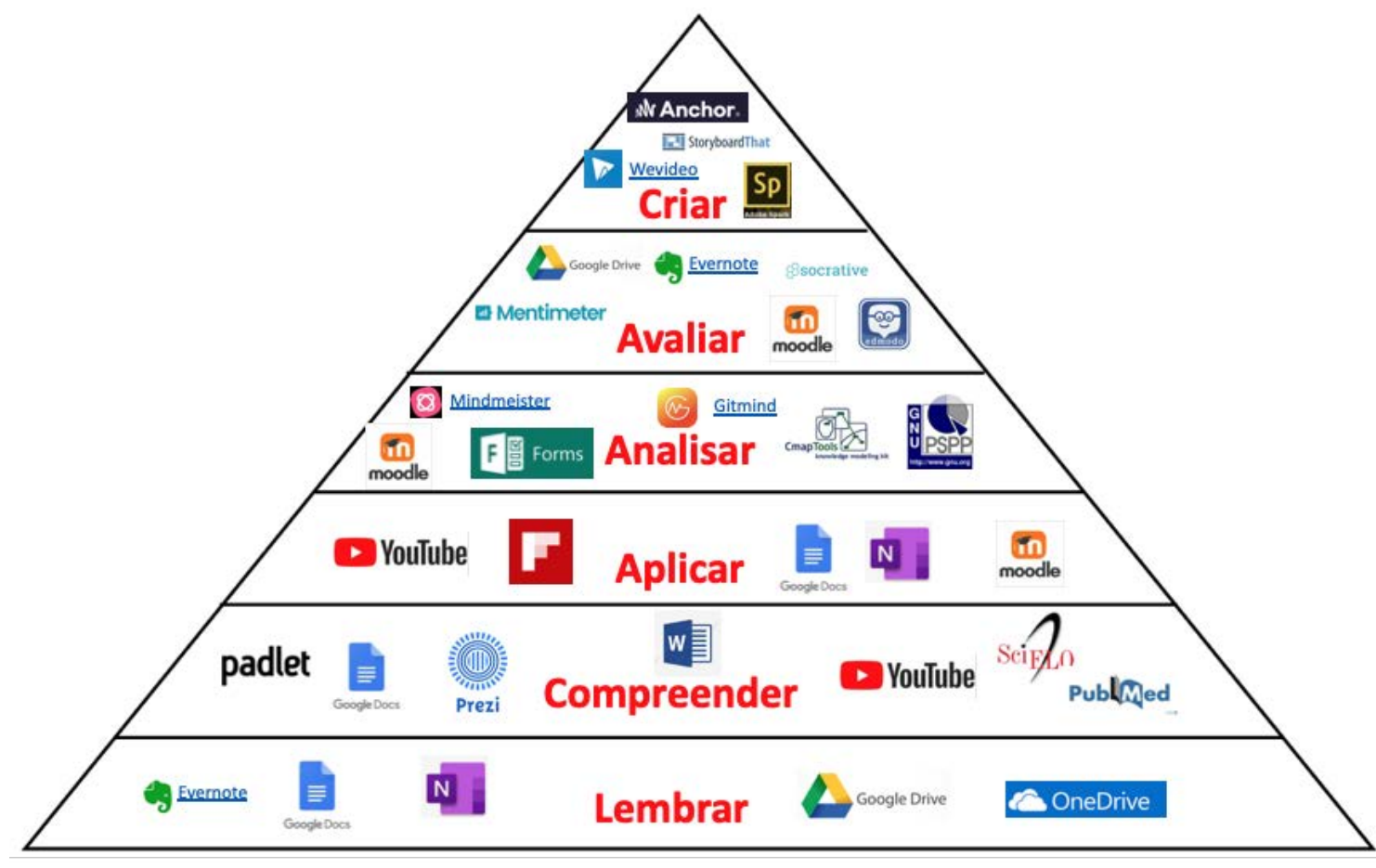

Fonte: Autores, 2021 


\section{ENSINO-APRENDIZAGEM CENTRADO NO ESTUDANTE NA ERA DIGITAL}

Da segunda metade do século passado aos dias de hoje observamos uma mudança progressiva do ensino centrado no professor para o aprendizado centrado no estudante, facilitando o que é definido como aprendizagem significativa ou profunda. A aprendizagem significativa é aquela que o estudante consegue incorporar o novo conhecimento a uma estrutura de conhecimento específica que faz parte integrante da sua estrutura cognitiva prévia ${ }^{26}$. Transportando os objetivos de aprendizagem para a era digital é importante elencar algumas habilidades necessárias básicas para utilização mais efetiva de estratégias e ferramentas nos ambientes virtuais de ensino-aprendizagem, conforme apresentado no "Conference Board of Canada" de $2014^{27}$ (Quadro 4). Isto demonstra a maior responsabilidade do estudante para com o seu aprendizado e indica um direcionamento aos professores na estruturação dos programas de ensino-aprendizagem. Outra iniciativa que afere as competências digitais de docentes e estudantes é o DigicompEdu, elaborada pela União Européia (https://ec.europa.eu/jrc/en/digcompedu).

\section{Quadro 4: Habilidades necessárias na era digital*}

\begin{tabular}{ll}
\hline Comunicação & $\begin{array}{l}\text { Além das habilidades tradicionais (ler, escrever e falar coerente e de manei- } \\
\text { ra clara) é preciso adicionar comunicação em mídias sociais }\end{array}$ \\
\hline $\begin{array}{l}\text { Capacidade de aprender de maneira } \\
\text { independente }\end{array}$ & $\begin{array}{l}\text { Responsabilidade para descobrir o que precisa saber e onde encontrar esse } \\
\text { conhecimento. Criação do hábito de aprendizado continuado por toda a vida }\end{array}$ \\
\hline Ética e responsabilidade & Necessário para construir confiança \\
\hline Trabalho em equipe e flexibilidade & Fundamental para as profissões da saúde \\
\hline $\begin{array}{l}\text { Habilidades de pensamento (pen- } \\
\text { samento crítico, resolução de pro- } \\
\text { blemas, criatividade, originalidade, } \\
\text { estratégia) }\end{array}$ & Capacidade de identificar as necessidades e encontrar soluções adequadas \\
\hline Habilidades digitais & $\begin{array}{l}\text { A maioria das atividades baseadas no conhecimento depende fortemente do } \\
\text { uso de tecnologia }\end{array}$ \\
\hline $\begin{array}{l}\text { Gestão do conhecimento } \\
\text { A mais abrangente de todas as habilidades: como encontrar, avaliar, anali- } \\
\text { sar, aplicar e disseminar informação, dentro de um determinado contexto. } \\
\text { Com volume e as rápidas mudanças que sofre o conhecimento (saber) é } \\
\text { impossível dominar todo o conteúdo }\end{array}$ \\
${ }^{*}$ Adaptado de Teaching in a Digital Age: Guidelines for designing teaching and learning an open book from A.W. (Tony) Bates)
\end{tabular}

As habilidades "digitais" listadas e os conceitos de ER e EaD permitem entender um pouco mais o contexto do desenvolvimento da sala de aula invertida e do ensino híbrido, ambos amplamente utilizados na sua versão tradicional ou modificada, e que se tornaram a base da maior parte dos programas de ensino durante a pandemia. O conceito de ensino híbrido está baseado na programação e estruturação intencional das atividades com objetivos claros para cada atividade, incluindo atividades online e face a face, atividades à distância (online ou não) e presenciais, misturando ou combinando tecnologias instrucionais com tarefas que simulam o trabalho, bem como combinando computadores com métodos tradicio- nais de ensino ${ }^{28}$. Já a sala de aula invertida tem fases bem definidas cujo racional é que a aula (ou melhor, a atividade síncrona) é o momento em que o professor e estudantes trabalham em atividades de análise, avaliação e criação capazes de produzir aprendizagem significativa ("deep learning" ${ }^{29}$. As fases da sala de aula invertida são: a) fase pré-classe (à distância), onde os estudantes desenvolvem tarefas utilizando material disponibilizado pelo professor (textos, videoaulas gravadas pelos docentes ou já disponíveis na rede, outros); b) fase síncrona (presencial ou online), onde ocorre a checagem da aprendizagem dos conceitos essenciais, onde novamente os estudantes desenvolvem tarefas, respondem questio- 
nários, resolvem problemas clínicos que os desafiem a utilizar o que aprenderam (individualmente ou em pequenos grupos), com o professor facilitando o aprendizado e esclarecendo dúvidas; e c) fase de síntese final da atividade, onde é realizada checagem do conhecimento, geralmente com avaliação formativa. Quando necessário pode-se propor complementações ${ }^{29}$.

Cabe salientar que a responsabilidade do professor nas estratégias de ensino centradas no estudante não é menor ou secundária. E isto não é diferente para o ER e EaD. Garantir que os estudantes alcancem as competências desejadas é tarefa comum a qualquer processo sério de ensino e aprendizagem. Neste aspecto é interessante perceber que a situação emergencial determinada pela pandemia fez com que muitos docentes tivessem que rever e repensar suas práticas objetivando a migração para o ambiente de ER e de EaD, trazendo desconforto inerente à mudança. Para muitos isto representou uma oportunidade de revisão também dos objetivos de aprendizado e do planejamento. Observando experiências e relatos de muitos educadores, publicados em vários formatos, no último ano, é possível listar várias dicas e estratégias de melhores práticas para o planejamento e a aplicação de atividades de ensino e aprendizagem no ER e EAD (Quadro 5). Embora não se trate de lista definitiva, espera-se que sirva de base para aqueles que estão iniciando ou que queiram checar suas atividades como docente neste momento de transformação ${ }^{30}$ e oportunidades ${ }^{31}$.

\section{Quadro 5: Dicas e estratégias para planejamento e aplicação de atividades de ensino e aprendizagem no ER e EAD}

\begin{tabular}{|c|c|}
\hline Dicas e estratégias & Comentários \\
\hline $\begin{array}{l}\text { Estabeleça sua presen- } \\
\text { ça imediatamente }\end{array}$ & $\begin{array}{l}\text { Muito importante para que os estudantes percebam que o bloco ou atividade tem um } \\
\text { responsável, aumentando a sensação de segurança, de seriedade e de expectativa de } \\
\text { aproveitamento }\end{array}$ \\
\hline $\begin{array}{l}\text { Defina expectativas cla- } \\
\text { ras para o curso, bloco } \\
\text { ou atividade }\end{array}$ & $\begin{array}{l}\text { A apresentação dos objetivos seguida da descrição do que está planejado, com detalha- } \\
\text { mento do tempo para cada atividade é fundamental para reduzir a ansiedade e conseguir } \\
\text { um bom desenvolvimento do processo }\end{array}$ \\
\hline $\begin{array}{l}\text { Esteja disponível (abra } \\
\text { mais de um canal de } \\
\text { comunicação) }\end{array}$ & $\begin{array}{l}\text { Os ambientes virtuais de aprendizagem (AVAs) contam com recursos de comunicação, } \\
\text { como aplicativos de mensagens, chat e fóruns. No entanto, é interessante ter pelo menos } \\
\text { um canal fora do ambiente (WhatsApp, Messenger...), considerando possíveis imprevistos } \\
\text { com a rede de internet e com o próprio AVA. }\end{array}$ \\
\hline Use recursos online & $\begin{array}{l}\text { Existe enorme quantidade de recursos online disponíveis que permitem tanto auxiliar } \\
\text { na disponibilização de material instrucional, bem como na aplicação de estratégias (ex.: } \\
\text { questionários pré-teste com devolutiva gravada instantânea) }\end{array}$ \\
\hline $\begin{array}{l}\text { Não fique centrado em } \\
\text { aulas formais }\end{array}$ & $\begin{array}{l}\text { Aulas formais devem ser transformadas em videoaulas curtas (entre } 7 \text { e } 15 \text { min de dura- } \\
\text { ção), seguindo as melhores práticas para produção deste tipo de material: contextualiza- } \\
\text { ção, objetivos claros (e essenciais), apresentação final dos pontos mais importantes }\end{array}$ \\
\hline $\begin{array}{l}\text { Deixe suas tarefas } \\
\text { claras }\end{array}$ & $\begin{array}{l}\text { Programe tarefas com objetivos claros e direcionados, seguindo o alinhamento adequa- } \\
\text { do para a aquisição de competência elencada. Tenha claro a estimativa de tempo para } \\
\text { conclusão da(s) tarefa(s) }\end{array}$ \\
\hline $\begin{array}{l}\text { Forneça devolutiva con- } \\
\text { tínua } \mathrm{e}(\mathrm{ou}) \text { constante }\end{array}$ & $\begin{array}{l}\text { As devolutivas devem estar presentes nas atividades síncronas ou assíncronas, sendo } \\
\text { facilitadas pelas plataformas online que permitem textos, imagens e vídeos explicativos. } \\
\text { Além disto, programe atividade síncrona para discussão, resolução de dúvidas e reforço } \\
\text { de pontos importantes }\end{array}$ \\
\hline $\begin{array}{l}\text { Conheça a sua platafor- } \\
\text { ma / domine a tecno- } \\
\text { logia }\end{array}$ & $\begin{array}{l}\text { Considerando a quantidade e complexidade dos recursos presentes nas plataformas é } \\
\text { importante testar e entender o modo de funcionamento de cada um, procurando aplicar } \\
\text { de maneira gradual até atingir proficiência e segurança. Para isto, sempre tenha uma } \\
\text { alternativa de atividade à mão }\end{array}$ \\
\hline $\begin{array}{l}\text { Crie um ambiente de } \\
\text { sala de aula online }\end{array}$ & $\begin{array}{l}\text { Existem várias soluções de videoconferência, com vários recursos comuns e outros parti- } \\
\text { cularizados, que atendem esta necessidade (os mais conhecidos são Zoom, Google Meet } \\
\text { e Mconf). Além da solução propriamente dita, deve-se ter a preocupação com o ambiente } \\
\text { de maneira mais ampla, de preferência acordando regras de trabalho em grupo (respeito, } \\
\text { cordialidade, liberdade, atenção e outras) }\end{array}$ \\
\hline
\end{tabular}


Interaja com seus alunos online

Seja organizado

A interação online depende da composição do grupo, mas muitas vezes é das tarefas mais difíceis. A principal estratégia é manter a abertura para perguntas e dúvidas, estimulando através de tarefas desenhadas para criar discussão sobre o tema. A interação não precisa ser necessariamente exclusivamente síncrona, sendo que a manutenção de vários canais para dúvidas faz parte da estratégia geral

O planejamento é extremamente importante, independentemente do formato (presencial, EaD ou ER), no entanto, a transição do presencial para o EaD ou ER requer e o mesmo tempo facilita uma maior organização. Criar o passo a passo das atividades se colocando no papel do estudante é de grande valia

Colabore com outros professores

A participação em discussões sobre estratégias de ensino e aprendizagem com colegas docentes sempre traz grande benefício para ampliar horizontes e compartilhar as meIhores práticas. Isto é potencializado quando existe uma comunidade de práticas para se inserir, maximizando o desenvolvimento profissional como professor

Reflita sobre as atividades e reveja o planejamento

Tenha um bom equilíbrio entre líder ativo e observador ativo

A reflexão ativa sobre a prática, considerando o que obteve sucesso e o que pode melhorar, deve ser uma constante. A avaliação discente e eventual acompanhamento de colega docente auxiliam sobremaneira esta análise

O acompanhamento do grupo e de cada indivíduo do grupo é muito importante para garantir um bom desenvolvimento do processo de ensino e aprendizagem. Alguns AVAs têm ferramentas que permitem acompanhar a realização das atividades pelos estudantes, abrindo oportunidade para comunicação ativa e efetiva ("está tudo certo?", "alguma dificuldade na tarefa?", "posso ajudar de alguma forma?")

Solicite devolutiva regu- A depender do plano de ensino é possível programar avaliações dos estudantes praticalar e fique atento para detectar mal entendidos

Espere o inesperado e permaneça flexível mente a cada atividade ou ao final de cada bloco. Esta é uma ferramenta poderosa para correção imediata de problemas ou mal entendidos

Estar preparado para remarcar ou trocar uma atividade devido à pane no sistema ou falta de energia que impeçam uma atividade online síncrona é o exemplo máximo desta questão e que mostra o grau de preparo que é preciso ter. Esteja pronto para propor remarcação ou enviar tarefa via outro canal de comunicação para cumprir com a meta traçada (parcial ou totalmente)

\section{REFERÊNCIAS}

1. Ellaway R, Masters K. e-Learning in Medical Education. Association for Medical Education in Europe (AMEE); 2008.

2. Gordon M, Patricio M, Horne L, Muston A, Alston SR, Pammi $M$, et al. Developments in medical education in response to the COVID-19 pandemic: A rapid BEME systematic review: BEME Guide No. 63. Med Teach. 2020;42(11):1202-15.

3. Lima L. MEC autoriza ensino a distância em cursos presenciais (http://portal.mec.gov.br/busca-geral/12-noticias/ acoes-programas-e-projetos-637152388/86441-mec-autoriza-ensino-a-distancia-em-cursos-presenciais). 2020.

4. Waseh S, Dicker AP. Telemedicine Training in Undergraduate Medical Education: Mixed-Methods Review. JMIR Med Educ. 2019;5(1):e12515.

5. JC DEG, Koppel PD, Kim SS, Park HK, Rushton S. Pedagogical foundations of cybercivility in health professions education: a scoping review. BMC Med Educ. 2021;21(1):79.

6. Hilty D, Chan S, Torous J, Luo J, Boland R. A Framework for Competencies for the Use of Mobile Technologies in Psychiatry and Medicine: Scoping Review. JMIR Mhealth Uhealth. 2020;8(2):e12229.
7. Silvennoinen P. Professional Master's Degree Students' Perceptions on the Changes Digitalisation Imposes on Counselling in the Social and Health Care Sector. Int J Environ Res Public Health. 2020;17(17).

8. BLASCHKE, L. M. Self-Determined Learning: designing for heutagogic learning environments. In: SPECTOR, $M$. J.; LOCKEE, B. B.; CHILDRESS, M. D. (Ed.). Learning, design, and technology: an international compendium of theory, research, practice, and policy. [S. I.]: Springer International Publishing, 2020.

9. Bollela VR, Machado JLM. O Currículo por Competências e sua Relação com as Diretrizes Curriculares Nacionais para a Graduação em Medicina. Revista Science in Health. $2010 ; 1: 126-42$.

10. Troncon LEdA. Estruturação de Sistemas para Avaliação Programática do Estudante de Medicina. Revista Brasileira de Educação Médica. 2016;40(1):30-42.

11. Troncon LEdA. Avaliação do estudante de medicina. Medicina (Ribeirão Preto). 1996;29(4):429-3.

12. Panúncio-Pinto MP, Troncon LEdA. Avaliação do estudante - aspectos gerais. Medicina (Ribeirão Preto). 2014;47(3):314-23.

13. Hope D, Davids V, Bollington L, Maxwell S. Candidates undertaking (invigilated) assessment online show no differences in performance compared to those undertaking assessment offline. Med Teach. 2021:1-14. 
14. Bouwmeester RA, de Kleijn RA, Freriksen AW, van Emst MG, Veeneklaas RJ, van Hoeij MJ, et al. Online formative tests linked to microlectures improving academic achievement. Med Teach. 2013;35(12):1044-6.

15. Mooney CJ, Peyre SE, Clark NS, Nofziger AC. Rapid transition to online assessment: Practical steps and unanticipated advantages. Med Educ. 2020;54(9):857-8.

16. Palmer E, Devitt P. The assessment of a structured online formative assessment program: a randomised controlled trial. BMC Med Educ. 2014;14:8.

17. Velan GM, Jones P, McNeil HP, Kumar RK. Integrated online formative assessments in the biomedical sciences for medical students: benefits for learning. BMC Med Educ. 2008;8:52.

18. Poot R, de Kleijn RA, van Rijen HV, van Tartwijk J. Students generate items for an online formative assessment: Is it motivating? Med Teach. 2017;39(3):315-20.

19. Harden RM, Laidlaw JM. Understanding basic educational principles. In: Harden RM, Laidlaw JM, editors. Essential Skills for a Medical Teacher. Third ed: Elsevier Limited; 2021. p. 148.

20. Miller GE. The assessment of clinical skills/competence/ performance. Acad Med. 1990;65(9 Suppl):S63-7.

21. Souza CdS, Iglesias AG, Pazin-Filho A. Estratégias inovadoras para métodos de ensino tradicionais - aspectos gerais. Medicina (Ribeirão Preto). 2014;47(3):284-92.

22. Peres CM, Suzuki KMF, Azevedo-Marques PMd. Recursos tecnológicos de apoio ao ensino na saúde. Medicina (Ribeirao Preto Online). 2015;48(3).

23. Ferreira, T. C., Ota, M. A., \& de Araujo Jr, C. F. (2021). Framework para o planejamento de aulas ativas nos espaços de aprendizagem online e presencial. Brazilian Journal of Development, 7(1), 2969-2979.
24. Churches A. Bloom's digital taxonomy. Australian School Library Association NSW Incorporated http://burtonslifelearningpbworkscom/f/BloomDigitalTaxonomy2001pdf. 2008.

25. Skiba DJ. Bloom's digital taxonomy and word clouds. Nurs Educ Perspect. 2013;34(4):277-80.

26. de Souza Lopes, L. A., \& da Silva Vieira, M. M. (2020). Digital Culture and Learning in Higher Education After COVID19: A Collaborative Approach in a Virtual Environment. European Journal of Education, 3(2), 103-116.

27. Bates AW. The skills needed in a digital age. In: Bates AW, editor. Teaching in a Digital Age: Guidelines for designing teaching and learning: BCcampus (https://open.umn.edu/opentextbooks/textbooks/221); 2015.

28. Liu Q, Peng W, Zhang F, Hu R, Li Y, Yan W. The Effectiveness of Blended Learning in Health Professions: Systematic Review and Meta-Analysis. J Med Internet Res. 2016;18(1):e2.

29. Tolks D, Schafer C, Raupach T, Kruse L, Sarikas A, Gerhardt-Szep $S$, et al. An Introduction to the Inverted/Flipped Classroom Model in Education and Advanced Training in Medicine and in the Healthcare Professions. GMS J Med Educ. 2016;33(3):Doc46.

30. Arco Bravo, I. D., Silva, P., \& Flores i Alarcia, Ò. (2021). University Teaching in Times of Confinement: The Light and Shadows of Compulsory Online Learning. Sustainability, 2021, vol. 13, núm. 1, p. 1-16.

31. Chao, T. N., Frost, A. S., Brody, R. M., Byrnes, Y. M., Cannady, S. B., Luu, N. N., ... \& Newman, J. G. (2021). Creation of an interactive virtual surgical rotation for undergraduate medical education during the COVID-19 pandemic. Journal of Surgical Education, 78(1), 346-350. 
Editor:

Prof. Dr. Marcelo Riberto

Recebido: $30 / 04 / 2021$

Aprovado: $13 / 07 / 2021$

(c) (i) Este é um artigo publicado em acesso aberto (Open Access) sob a licença Creative

sem restrições, desdé que o trabalho original seja corretamente citado. 